\title{
Bioelectrode based on reconstitution of a dye-linked L-proline dehydrogenase on gold electrode modified by PQQ and FAD
}

\author{
Yanhui Song ${ }^{1, a}$, Ningxin Wang ${ }^{1, b}$, Haitao Zheng ${ }^{1, c, *}$ and Shin-ichiro Suye ${ }^{2, d}$ \\ ${ }^{1}$ School of Environmental and Chemical Engineering, Tianjin Polytechnic University, Tianjin \\ 300387, China \\ ${ }^{2}$ Department of Applied Chemistry and Biotechnology, Graduate School of Engineering, University of \\ Fukui, Bunkyo 3-9-1, Fukui 910-8507, Japan \\ asongyanhui0317@sina.com, bningxin_wang@sina.com, czhenght999@hotmail.com, \\ dsuyeb10@u-fukui.ac.jp
}

Keywords: L-proline sensor, reconstitution of apo-enzyme, electron relay.

\begin{abstract}
An electrochemical sensor was developed by reconstituting pyrroloquinoline quinone (PQQ), flavin adenine dinucleotide (FAD) and L-proline dehydrogenase on gold electrode. Reversible redox peaks and low redox potential were obtained from cyclic voltammograms, proved that the attached PQQ and FAD accelerated electron communication between enzyme active site and gold electrode surface. It is expected that this reconstitution technology is suitable for the application of this modified electrode to bioanode.
\end{abstract}

\section{Introduction}

The effective electron communication between enzyme active site and solid electrode surface is of great importance for enzyme-based electrochemical devices [1], including biosensors, biofuel cell and etc. As the active sites are sealed deeply by the enzyme protein shell, various kinds of methods have been developed to improve and accelerate the electron transfer procedure [2], including the utilization of a numbers of electron mediators, and the combination of enzyme with nano-scale materials (nano metal particles or carbon nanotubes). On the other hand, researchers also tried the way to reconstitute the redox enzymes on electrode surface for both immobilization and electron relay.

In this work, the reconstitution technique was utilized for the immobilization and electron transfer of L-proDH Au electrode with covalently attached PQQ and FAD monolayer was used as the platform, on which an apo-L-proDH was immobilized based on the reconstitution of apo-enzyme on the FAD containing monolayer on the electrode. The redox potential of PQQ is ca. $-0.1 \mathrm{~V}$ vs. SCE, resulting a higher voltage output. Furthermore, the electron relay between FAD site and electrode will be accelerated efficiently as PQQ was covalently linked to FAD directly.

\section{Experimental}

Pyrroloquinoline quinone (PQQ), flavin adenine dinucleotide (FAD), 1-ethyl-3[3-dimethylaminopropyl] carbodiimide hydrochloride (EDC), cystamine were obtained from Wako Chemical In. (Tokyo, Japan), and N-hydroxysulfosuccinimide was purchase from sigma (USA). All the other chemicals were of analytical grade.

The Au electrodes were finely polished by $1 \mu \mathrm{m}$ and $0.05 \mu \mathrm{m}$ aluminum slurry, and scanned over the potential range of $+0.0 \mathrm{~V}$ to $+1.7 \mathrm{~V}$ (vs. $\mathrm{Ag} / \mathrm{AgCl}$ ) in $0.5 \mathrm{mM} \mathrm{H}_{2} \mathrm{SO}_{4}$ solution for about 30 minutes until the same voltammogram was obtained. After thoroughly rinsed by water, the cleaned electrode was immersed in a $20 \mathrm{mM}$ cystamine solution for $2 \mathrm{~h}$, followed by rinsed with water. The obtained cystamine modified electrode was referred as Au/cystamine.

The PQQ was immobilized on Au/cystamine by immersing the electrode in 10 mM HEPES buffer (pH 7.2) containing $3 \mathrm{mM}$ EDC, $3 \mathrm{mM}$ NHS and $1 \mathrm{mM}$ PQQ for $5 \mathrm{~h}$ under stirring, during which 
PQQ was covalently linked, and the electrode was referred as Au/cystamine/PQQ. The FAD moisture was further attached on the PQQ modified electrode by immersing Au/cystamine/PQQ in $10 \mathrm{mM}$ HEPES ( $\mathrm{pH}$ 7.2) containing $3 \mathrm{mM}$ EDC, $3 \mathrm{mM}$ NHS and $1 \mathrm{mM} F A D$ for $5 \mathrm{~h}$ under stirring, and the resulted electrode was referred as Au/cystamine/PQQ/FAD.

\section{Results and discussion.}
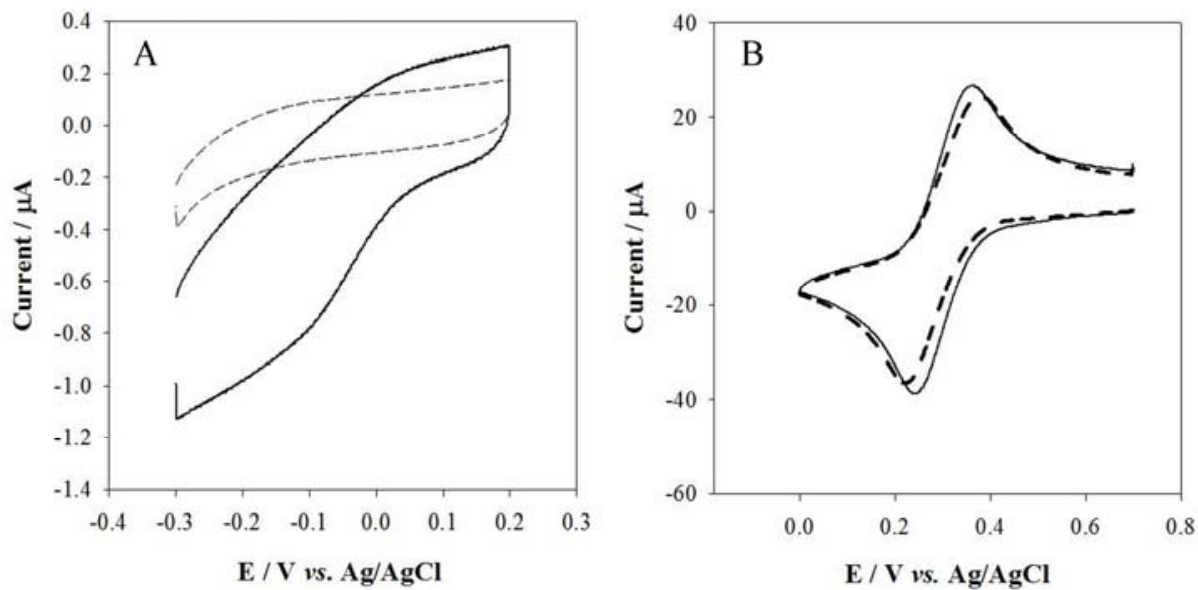

Fig. 1 Cyclic voltammograms of Au electrode before (solid line) and after (dash line) self-assembled monolayer of cystamine in $50 \mathrm{mM}$ PBS (pH 7.0) at a scan rate of $100 \mathrm{mV} \mathrm{s}-1(\mathrm{~A})$ and in $50 \mathrm{mM}$ PBS (pH 7.0) containing $2 \mathrm{mM} \mathrm{K3Fe}(\mathrm{CN}) 6$ and $0.8 \mathrm{M} \mathrm{KCl}$ at a scan rate of $400 \mathrm{mV} \mathrm{s}-1(\mathrm{~B})$.

The cyclic volgammographs of $\mathrm{Au}$ electrode before and after treatment of $20 \mathrm{mM}$ cystamine in air-saturated buffer solution are shown in Fig. 1A. It was found clearly that the basic current decreased $50 \%$ after the self-assembled monolayer of cystamine. The electrochemical reduction of trace oxygen appeared at ca. $-0.2 \mathrm{~V}$ on the bare electrode, and was blocked by the monolayer of cystamine.

The self-assembled monolayer of cystamine was also studied by using $\mathrm{K}_{3} \mathrm{Fe}(\mathrm{CN})_{6}$ as a probe, and the cyclic voltammographs of bare and cystamine-modified $\mathrm{Au}$ electrode in phosphate buffer containing $\mathrm{K}_{3} \mathrm{Fe}(\mathrm{CN})_{6}$ are shown in Fig. 1B. The redox peaks of $\mathrm{K}_{3} \mathrm{Fe}(\mathrm{CN})_{6}$ became a little irreversible only when the scan rate was larger than $400 \mathrm{mV} \mathrm{s}^{-1}$. The reason might be that the carbon chain in cystamine is short, and the thiol molecules could not form a very compact film on electrode surface. This phenomena was also illustrated by Kim [3], who investigated aminoalkanethiol monolayer on $\mathrm{Au}$ electrode with different carbon chain. On the other hand, the protonated amino groups on the monolayer carried positive charges, which attracted and concentrated $\mathrm{Fe}(\mathrm{CN})_{6}{ }^{3-}$ based on the electrostatistic force between opposite charges[4].

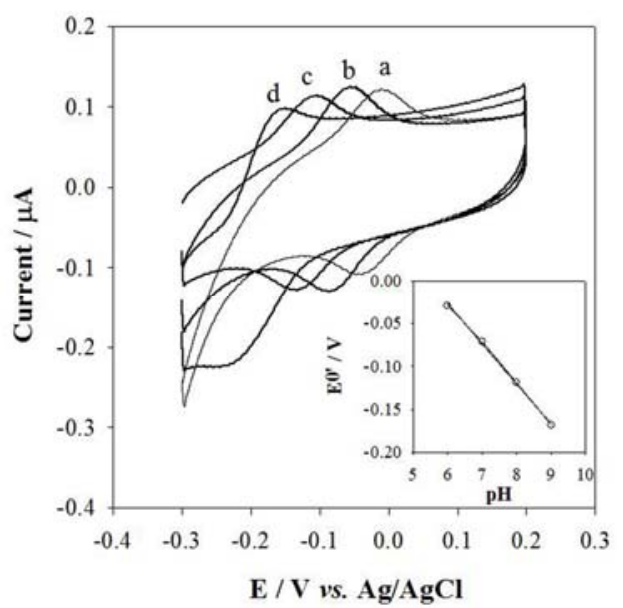

Fig. 2 Cyclic voltammograms of Au/cystamine/PQQ in pH 6.0 (a), 7.0 (b), 8.0 (c) and 9.0 (d) of 50 mM PBS at a scan rate of $100 \mathrm{mV} \mathrm{s}-1$. Insert: The dependence of E0' on buffer $\mathrm{pH}$. 
The electrochemical behavior of Au/cystamine/PQQ electrode in phosphate buffers with different $\mathrm{pH}$ was studied and shown in Fig. 2. The redox peak potentials were dependent on buffer $\mathrm{pH}$, and a linear relationship between the formal potential $E^{0^{\prime}}\left(E^{0^{\prime}}=\left(E_{p a}+E_{p c}\right) / 2\right)$ and buffer $\mathrm{pH}$ was found (shown in the insert figure), and an increase of one $\mathrm{pH}$ unit resulted in the shift of redox potential of ca. $-40 \mathrm{mV}$, which suggested a two electrons and one proton process during this $\mathrm{pH}$ range. This phenomena was also pointed out by Katz[5].

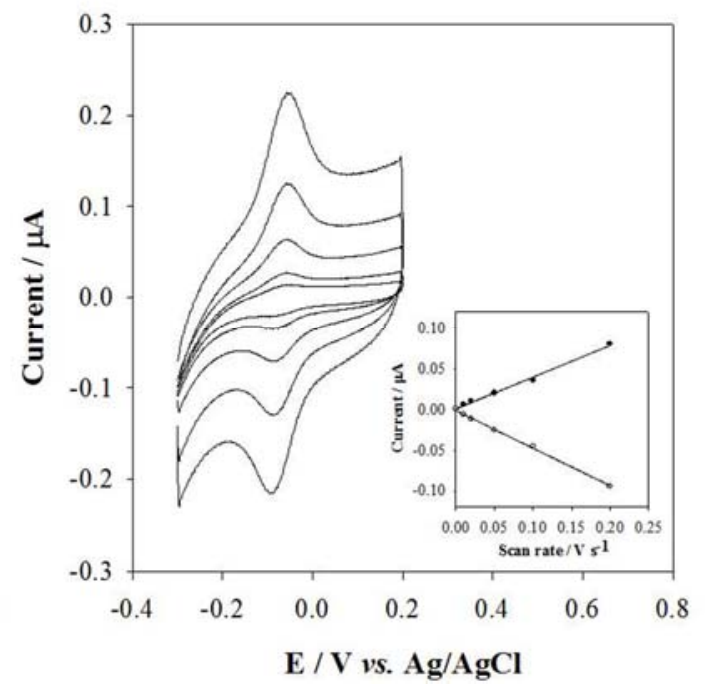

Fig. 3 Cyclic voltammograms of Au/cystamine/PQQ in $50 \mathrm{mM}$ PBS (pH 7.0) at different scan rate $(10,20,50,100$ and $200 \mathrm{mV} \mathrm{s}-1$ from inner to outer). Insert: Influence of scan rate on Ipa and Ipc.

The cyclic voltammographs of Au/cystamine under different scan rates are plotted in Fig. 3. A well-defined redox peaks were found clearly, and the peak-to-peak separation was ca. $39 \mathrm{mV}$. Both of anodic and cathodic peak currents increased linearly proportional to scan rates (inserted figure of Fig. 3 ), suggesting a diffusion-free electrochemical process. The surface coverage $(\Gamma)$ was estimated at ca. $2.1 \times 10^{-11} \mathrm{~mol} \mathrm{~cm}^{-2}$ by calculating the anodic peak area at the scan rate of $20 \mathrm{mV} \mathrm{s}^{-1}$.

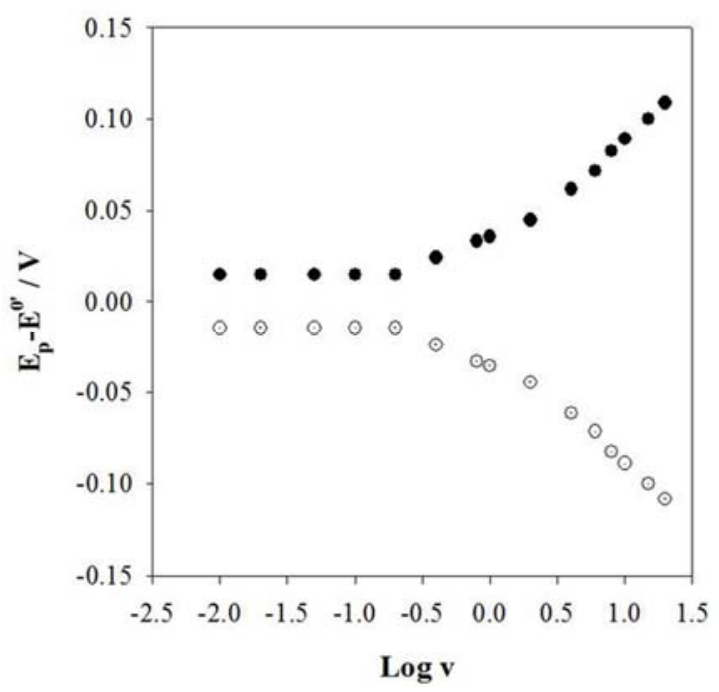

Fig. 4 Plots of anodic and cathodic peak potential against the logarithm of scan rate. All experiments were carried out on Au/cystamine/PQQ in 50 mM PBS (pH 7.0).

In order to analyze the electron-transfer kinetics, cyclic voltammograph of $\mathrm{Au} /$ cystamine/PQQ at higher scan rates were also measured, and the plot of peak-to-peak separation verse Logv is shown in Fig. 4. Because both the anodic and cathodic peaks shifted symmetrically in positive and negative direction, the electron transfer coefficient $(\alpha)$ should be equal to nearly 0.5 . When the scan rate was larger than $400 \mathrm{mV} \mathrm{s}^{-1}$, the peak separation $(\Delta E)$ increased dramatically, suggesting a more and more irreversible electron-transfer kinetic occurred. According to Laviron equation for an irreversible cyclic voltammograph $(\Delta E>230 \mathrm{mV})$, the apparent rate constant $k_{s}$ can be obtained at $c a .7 .8 \mathrm{~s}^{-1}$, by 
extrapolating the linear portion of the $\Delta E$ verse $\log v$ curves (scan rate between 6 and $20 \mathrm{~V} \mathrm{~s}^{-1}$ ) in Fig. 4.

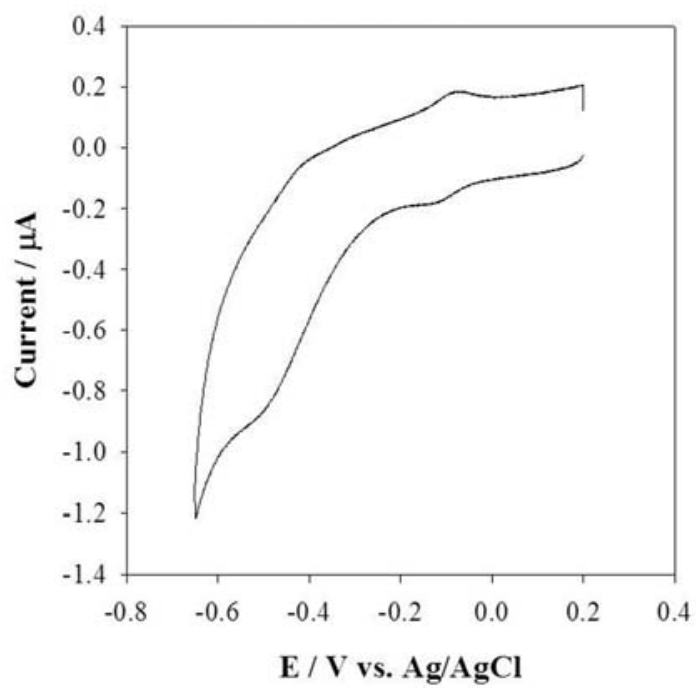

Fig. 5 Cyclic voltammogram of Au/cystamine/PQQ/FAD in $50 \mathrm{mM}$ PBS (pH 7.0) at a scan rat of 100 $\mathrm{mV} \mathrm{s}-1$.

The FAD molecules were further immobilized on Au/cystamine/PQQ electrode by the same procedure under the help of EDC and NHS, and its electrochemical behavior was measured by cyclic voltammetry, shown in Fig. 5. It was found that a pair of small redox appeared at around $-0.41 \mathrm{~V}$, which owns to the redox process of immobilized FAD, but the surface immobilization amount is very small.

\section{Conclusion}

A novel modified electrode was successfully fabricated by reconstituting PQQ, FAD and L-proDH on gold electrode. The developed electrode exhibited good electrochemical properties, which confirms that electrochemical communication has been achieved between immobilized enzyme and electrode by reconstitution technology. So this fabricated electrode can be used in practical detection owns to its stability and electrochemical activity.

\section{References}

[1]. I. Willner, E. Katz, and B. Willner, Electrical contact of redox enzyme layers associated with electrodes: Routes to amperometric biosensors, Electroanalysis, Vol. 9(1997), p. 965-977.

[2]. W. Schuhmann, Amperometric enzyme biosensors based on optimised electron-transfer pathways and non-manual immobilisation procedures, Rev. Mol. Biotechnol., Vol. 82(2002), p. 425-441.

[3]. W. Park, J. Ahmed, and S. Kim, Heterogeneous electron-transfer kinetics for PQQ covalently attached to aminoalkanethiol monolayers on gold, Colloid Surf. B-Biointerfaces, Vol. 68(2009), p. 120-124.

[4]. S. Sarkar and S. Sampath, Stepwise assembly of acceptor-Sigma spacer-donor monolayers: Preparation and electrochemical characterization, Langmuir, Vol. 22(2006), p. 2388-3395.

[5]. E. Katz, D. D. Schlereth, and H.-L. Schmidt, Electrochemical study of pyrroloquinoline quinone covalently immobilized as a monolayer onto a cystamine-modified gold electrode, J. Electroanal. Chem., Vol. 367(1994), p. 59-70. 